\title{
Idiopathic localized lipodystrophy
}

INSERM

\section{Source}

INSERM. (1999). Orphanet: an online rare disease and orphan drug data base. Idiopathic localized lipodystrophy. ORPHA:90158

Idiopathic localized lipodystrophy is a rare, acquired, localized lipodystrophy characterized by asymptomatic, well-demarcated, depressed, lipoatrophic lesions of variable size, with normal overlying skin without antecedent inflammation or a known identifiable cause (autoimmune disease, drug injection, injury, etc). 